\title{
About the Relationship between Election Problem and Failure Detector in Asynchronous Distributed Systems
}

\author{
Sung-Hoon Park \\ Dept. of Computer Science, NamSeoul University, Chung-Nam 330-800, Korea \\ spark@nsu.ac.kr
}

\begin{abstract}
This paper is about the relationship between Election problem and Failure Detector in asynchronous distributed systems. We first discuss the relationship between the Election problem and the Consensus problem in asynchronous distributed systems with unreliable failure detectors. Chandra and Toueg have stated that Consensus is solvable in asynchronous systems with unreliable failure detectors. But, in contrast to the Consensus problem, the Election problem is impossible to solve with unreliable failure detectors even with a single crash failure. More precisely, the weakest failure detector that is needed to solve this problem is a Perfect Failure Detector, which is strictly stronger than the weakest failure detector that is needed to solve Consensus.
\end{abstract}

\section{Introduction}

To elect a Leader (or Coordinator) in a distributed system, an agreement problem must be solved among a set of participating processes. This problem, called the Election problem, requires the participants to agree on only one leader in the system [1]. The problem has been widely studied in the research community $[2,3,4,5,6]$. One reason for this wide interest is that many distributed protocols need an election protocol.

The Election problem is described as follows. At any time, there is at most one process that considers itself a leader and all other processes consider it as to be their only leader. If there is no leader, a leader is eventually elected.

Consensus and Election are similar problems in that they are both agreement problems. The so-called FLP impossibility result, which states that it is impossible to solve any non-trivial agreement in an asynchronous system even with a single crash failure, applies to both problems [7]. The starting point of this paper is the fundamental result of Chandra and Toueg [8], which states that Consensus is solvable in asynchronous systems with unreliable failure detectors.

An interesting question is then whether the Election problem can also be solved in asynchronous systems with unreliable failure detectors. The answer to this question is "No". This means that the Election problem is harder than the Consensus problem. However, in contrast to initial intuition, the reason Election is harder than Consensus is not upon its Liveness condition. The difficulty in solving Election is actually upon its Safety condition (all the processes connected the system never disagree on the leader). This condition requires precise knowledge about failures which unreliable 
failure detectors cannot provide. More precisely, the weakest failure detector that is needed to solve this problem is a Perfect Failure Detector, which is strictly stronger than the weakest failure detector that is needed to solve Consensus.

The rest of the paper is organized as follows. Section 2 describes motivations and the related works. In Section 3 we describe our system model. In Section 4 and 5 we show that the weakest failure detector for solving Election is a Perfect failure Detector. Finally, Section 6 summarizes the main contributions of this paper and discusses related and future work.

\section{Motivations and Related Works}

In recent years, several paradigms have been identified to simplify the design of faulttolerant distributed applications in a conventional static system. Election is among the most noticeable, particularly since it is closely related to group communication, which (among other uses) provides a powerful basis for implementing active replications.

It was shown in [7] that the Consensus problem cannot be solved in an asynchronous system if even a single crash failure can occur. The intuition behind this widely cited result is that in an asynchronous system, it is impossible for a process to distinguish between another process that has crashed and one that is merely very slow. The consequences of this result have been enormous, because most real distributed systems today can be characterized as asynchronous, and Consensus is an important problem to be solved if the system is to tolerate failures.

As a result, the Consensus problem has frequently been used as a yardstick of computability in asynchronous fault-tolerant distributed systems. That means that if any problem is harder than Consensus, it also cannot be solved in asynchronous systems.

The asynchronous model of computation is especially popular in practice because unpredictable workloads are sources of asynchrony in many real systems. Therefore rendering any synchrony assumption is valid only probabilistically. Thus, the impossibility of achieving Consensus reveals a serious limitation of this model for fault-tolerant applications such as the Election problem. Because Consensus is such a fundamental problem, researchers have investigated various ways of circumventing the impossibility.

Actually, the main difficulty in solving such a problem in presence of process crashes lies in the detection of crashes. As a way of getting around the impossibility of Consensus, Chandra and Toug extended the asynchronous model of computation with unreliable failure detectors and showed that the Consensus problem is solvable even with unreliable failure detectors [10].

We are not the first to show that there are problems harder than Consensus. The first such result that we are aware of is [11] in which the authors show that NonBlocking Atomic Commitment (NB-AC) cannot be implemented with the weakest failure detector that can implement Consensus. This problem arises when transactions update data in a distributed system and the termination of transactions should be coordinated among all participants if data consistency is to be preserved even in the presence of failures [12]. It resembles the Election problem in that NB-AC is harder than Consensus. 
To solve the NB-AC problem with an unreliable failure detector, they propose Non-Blocking Weak Atomic Commitment (NB-WAC) protocol and show that a failure detector weaker than a Perfect Failure Detector is strong enough to solve NonBlocking Weak Atomic Commitment (NB-WAC).

\section{Model and Definitions}

Our model of asynchronous computation with failure detection is the one described in [10]. In the following, we only recall some informal definitions and results that are needed in this paper.

\subsection{Processes}

We consider a distributed system composed of a finite set of processes $\Omega=\left\{p_{1}, p_{2}, . ., p_{n}\right\}$ completely connected through a set of channels. Communication is by message passing, asynchronous and reliable. Processes fail by crashing; Byzantine failures are not considered.

Asynchrony means that there is no bound on communication delays or process relative speeds. A reliable channel ensures that a message, sent by a process $p_{i}$ to a process $p_{j}$, is eventually received by $p_{j}$ if $p_{i}$ and $p_{j}$ are correct (i.e. do not crash).

To simplify the presentation of the model, it is convenient to assume the existence of a discrete global clock. This is merely a fictional device inaccessible to processes. The range of clock ticks is the set of natural numbers. A history of a process $p_{i} \in \Omega$ is a sequence of events $h_{i}=e_{i}^{0} \cdot e_{i}^{l} \cdot e_{i}^{2} \cdots e_{i}^{k}$, where $e_{i}^{k}$ denotes an event of process $p_{i}$ occurred at time $k$. Histories of correct processes are infinite. If not infinite, the process history of $p_{i}$ terminates with the event $\operatorname{crash}_{\mathrm{i}}{ }^{\mathrm{k}}$ (process $p_{i}$ crashes at time $k$ ). Processes can fail at any time, and we use $f$ to denote the number of processes that may crash. We consider systems where at least one process correct (i.e. $f$. $|\Omega|$ ).

A failure detector is a distributed oracle which gives hints on failed processes. We consider algorithms that use failure detectors. An algorithm defines a set of runs, and a run of algorithm $A$ using a failure detector $D$ is a tuple $R=\langle F, H, I, S, T\rangle: I$ is an initial configuration of $A ; S$ is an infinite sequence of events of $A$ (made of process histories); $T$ is a list of increasing time values indicating when each event in $S$ occured; $F$ is a failure pattern that denotes the set $F(t)$ of processes that have crashed at any time $t ; H$ is a failure detector history, which gives each process $p$ and at any time $t$, a (possibly false ) view $H(p, t)$ of the failure pattern: $H(p, t)$ denotes a set of processes, and $q \in H(p, t)$ means that process $p$ suspects process $q$ at time $t$.

\subsection{Failure Detector Classes}

Failure detectors are distributed oracles related to the detection of failures. A failure detector of a given class is a device that gives hints on a set of processes that it suspects to have crashed. 
The Oracle notion has first been introduced as a language whose words can be recognized in one step from a particular state of a Turing machine [13,14]. The main characteristic of such oracles is to hide a sequence of computation steps in a single step (they may also hide an uncomputable function). They have been used to provide a hierarchy of problems. Hence the Oracle notation is related to the detection of failures. These oracles do not change the pattern of failures that affect the execution in which they are used. The main characteristic of such oracles is not related to the number of computation steps they hide, but to the guess they provide about failures.

Failure detectors are abstractly characterized by completeness and accuracy properties [10]. Completeness characterizes the degree to which crashed processes are permanently suspected by correct processes. Accuracy restricts the false suspicions that a process can make.

Two completeness properties have been identified. Strong Completeness, i.e. there is a time after which every process that crashes is permanently suspected by every correct process, and Weak Completeness, i.e. there is a time after which every process that crashes is permanently suspected by some correct process.

Four accuracy properties have been identified. Strong Accuracy, i.e. no process is never suspected before it crashes. Weak Accuracy, i.e. some correct process is never suspected. Eventual Strong Accuracy (.Strong ), i.e. there is a time after which correct processes are not suspected by any correct process; and Eventual Weak Accuracy (.Weak ), i.e. there is a time after which some correct process is never suspected by any correct process. A failure detector class is a set of failure detectors characterized by the same completeness and the same accuracy properties (Fig. 1).

For example, the failure detector class $P$, called Perfect Failure Detector, is the set of failure detectors characterized by Strong Completeness and Strong Accuracy. Failure detectors characterized by Strong Accuracy are reliable: no false suspicions are made. Otherwise, they are unreliable.

\begin{tabular}{|c|c|c|c|c|}
\hline \multirow{2}{*}{ Completeness } & \multicolumn{4}{|c|}{ Accuracy } \\
\cline { 2 - 5 } & Strong & Weak & .Strong & .Weak \\
\hline Strong & $P$ & $S$ &.$P$ &.$S$ \\
Weak & $Q$ & $W$ &.$Q$ &.$W$ \\
\hline
\end{tabular}

Fig. 1. Failure detector classes. A failure detector class is a set of failure detectors characterized by the same completeness and the same accuracy properties.

For example, failure detectors of $S$, called Strong Failure Detector, are unreliable, whereas the failure detectors of $P$ are reliable.

\subsection{Reducibility and Transformation}

The notation of problem reduction first has been introduced in the problem complexity theory [14], and in the formal language theory [13]. It has been also used in the distributed computing $[15,16]$. We consider the following definition of problem reduction. An algorithm $A$ solves a problem $B$ if every run of $A$ satisfies the 
specification of $B$. A problem $B$ is said to be solvable with a class $C$ if there is an algorithm which solves $B$ using any failure detector of $C$. A problem $B_{l}$ is said to be reducible to a problem $B_{2}$ with class $C$, if any algorithm that solves $B_{2}$ with $C$ can be transformed to solve $B_{l}$ with $C$. If $B_{1}$ is not reducible to $B_{2}$, we say that $\mathrm{B}_{l}$ is harder than $B_{2}$.

A failure detector class $C_{1}$ is said to be stronger than a class $C_{2}$, (written $C_{1} \geq C_{2}$ ), if there is an algorithm which, using any failure detector of $C_{l}$, can emulate a failure detector of $C_{2}$. Hence if $C_{1}$ is stronger than $C_{2}$ and a problem B is solvable with $C_{2}$, then $B$ is solvable with $C_{l}$. The following relations are obvious: $\mathrm{P} \geq \mathrm{Q}, \mathrm{P} \geq \mathrm{S}, . \mathrm{P} \geq$.Q, $. \mathrm{P} \geq . \mathrm{S}, \mathrm{S} \geq \mathrm{W}, . \mathrm{S} \geq \mathrm{W}, \mathrm{Q} \geq \mathrm{W}$, and $. \mathrm{Q} \geq \mathrm{W}$. As it has been shown that any failure detector with Weak Completeness can be transformed into a failure detector with Strong Completeness [10], we also have the following relations: $\mathrm{Q} \geq \mathrm{P}, \mathrm{Q} \geq \mathrm{P}, \mathrm{W} \geq \mathrm{S}$ and .W $\geq$.S. Classes $\mathrm{S}$ and .P are incomparable.

\subsection{The Election Problem}

The Election problem is described as follows: At any time, at most one process considers itself the leader, and at any time, if there is no leader, a leader is eventually elected. More formally, the Election Problem is specified by the following two properties:

- Safety: All processes connected the system never disagree on a leader.

- Liveness: All processes should eventually progress to be in a state in which all processes connected to the system agree to the only one leader.

\subsection{The Consensus Problem}

In the Consensus problem (or simply Consensus), every participant proposes an input value, and correct participant must eventually decide on some common output value $[9,17]$. Consensus is specified by the following conditions.

- Agreement: no two correct participant decide different values;

- Uniform-Validity: if a participant decides $v$, then $v$ must have been proposed by some participant;

- Termination: every correct participant eventually decide.

Chandra and Toueg have stated the following two fundamental results [8]:

1. If $f<|\Omega|$, Consensus is solvable with either $S$ or $W$.

2. If $f<\lceil|\Omega| / 2\rceil$, Consensus is solvable with either.$S$ or.$W$. 


\section{Impossibility of Solving the Election Problem with Unreliable Failure Detectors}

In this section, we show that the Election problem is not solvable in asynchronous systems with unreliable failure detectors. This impossibility result holds even with the assumption that at most one process may crash. Though a Strong Failure Detector is sufficient to solve Consensus, it is not sufficient to solve Election. More precisely, we show that if $f>0$, Election can not be solved with either.$P$ or $S$.

Theorem 1. If $f>0$, Election can not be solved with either.P or $S$.

PROOF (by contradiction). Consider a failure detector $D$ of .P (respectively of $S$ ). We assume for a contradiction that there exists a deterministic election protocol $E$ that can be combined with a failure detector $D$ such that $E+D$ is also an election protocol. Consider an algorithm $A$ combined with $E+D$ which solves Election and a run $R=<$ $F, H_{D}, I, S, T>$ of $A$. We assume that only two processes $P_{i}$ and $P_{j}$ are correct. Consider that $P_{i}$ is a leader at time $\left(R, t_{0}\right)$. At time $\left(R, t_{l}\right)$ where $t_{l}>t_{0}$, the process $P_{j}$ sends a message to confirm whether the leader is alive. At time $\left(R, t_{2}\right)$ where $t_{2}>t_{1}$, the process $P_{i}$ sends a reply message to the process $P_{j}$. But at time $\left(R, t_{3}\right)$ where $t_{3}>t_{l}$, $P_{j}$ falsely suspects other process $P_{i}$ by the Weak accuracy property of the unreliable failure detector $D$ in some run. At time $\left(R, t_{4}\right)$ where $t_{4}>t_{3}, P_{j}$ considers itself a leader by delaying the receipt of the reply message sent by $P_{i}$ until $t_{5}$, where $t_{5}>t_{4}$. Thus at time $\left(R, t_{5}\right)$ both $P_{i}$ and $P_{j}$ consider themselves the leader, violating the assumption that $A$ is an election protocol. But after time $t_{5}$, all the processes except $P_{i}$ and $P_{j}$ are suspected. Hence there is a time after which every process that crashes is permanently suspected by every correct process. So $H_{D}$ satisfies Strong Completeness.

Consider Accuracy.

- If $D$ is of class .P, $H_{D}$ satisfies Eventual Strong Accuracy, i.e. there is a time after which correct participants are never suspected by any correct participant. As $P_{i}$ and $P_{j}$ are never suspected after time $t_{5}$ in $H_{D}$, then $H_{D}$ satisfies Eventual Strong Accuracy.

- If $D$ is of class $S, H_{D}$ satisfies Weak Accuracy, i.e. some correct participant is never suspected in $H_{D}$. As $P_{j}$ is never suspected by the correct process $P_{i}, H_{D}$ satisfies Weak accuracy.

- This is a contradiction.

By the relation between failure detector classes, we have the following Corollary.

Corollary 1. If $>0$, Election is not solvable with either. $Q, S, W$ or .W.

\section{The Weakest Failure Detector for Solving the Election Problem}

In the previous section, we showed that the Election problem is not solvable in asynchronous systems with unreliable failure detectors. Then, what is the weakest failure detector that is needed to solve this problem in asynchronous distributed 
systems? In this section, as the answer to this question, we show that a Perfect Failure Detector is the weakest failure detector for solving Election.

Theorem 2. Iff $>0$, a failure detector of class $Q$ is sufficient to solve Election.

PROOF: The Election problem can be solved using an election protocol $E$ combined with the following algorithm $A$ and a failure detector $D$ belonging to class $Q$ :

1. Each process has a unique ID number that is known by all processes a priority.

2. The leader is initially the process with the lowest ID number.

3. If a process detects a failure, it broadcasts this information to all other processes. Upon receiving such a message, the receiver detects the failure.

4. When a process detects the failure of all processes with lower ID numbers, then that process becomes the leader.

The proof that the protocol $E$ satisfies Election is as followings.

- Safety (proof by contradiction). The election protocol $E$ starts only when the current leader has failed by the strong Accuracy property of $D$ which belongs to the class $Q$ (line 3). Assume that the current leader has crashed at time $\left(R, t_{1}\right)$ in some run $R$ of the protocol $E$. We assume for the contradiction that two processes, $P_{i}$ and $P_{j}\left(P_{i} \neq P_{j}\right)$ are elected as leaders at time $\left(R, t_{5}\right)$, where $t_{5}>t_{l}$. To be elected as a leader, they must have detected the failure of all processes with lower ID numbers (line 4). That means that $P_{i}$ and $P_{j}$ have detected the failure of all processes with lower ID numbers at a time $\left(R, t_{3}\right)$ and at a time $\left(R, t_{4}\right)$ respectively, where $t_{1}<t_{3}, t_{4}$ $<t_{5}$. As two processes are different each other $\left(P_{i} \neq P_{j}\right)$, at least one of them has a lower ID number (line 1). Thus, one process falsely suspected the other. But it is contradiction to the assumption that a failure detector $D$ of class $Q$ has the strong Accuracy property, i.e. no process is never suspected before it crashes.

- Liveness. In case of the current leader's failure at time $\left(R, t_{1}\right)$ in some run of $E$, some correct process eventually detects the leader's crash by the Weak Completeness property of the failure detector $D$ of class $Q$ (i.e. there is a time after which every process that crashes is permanently suspected by some correct process ) and broadcasts this information at time $\left(R, t_{2}\right)$ where $t_{2}>t_{l}$ (line 3 ). By the reliable channel assumption, every correct process eventually receives the information and starts to detect the failures of all processes with lower ID (line 3). Because the process detected a failure broadcasts this information to all other processes (line 4$)$, every correct process eventually suspects every failed process. Thus at time $\left(R, t_{3}\right)$ where $t_{3}>t_{2}$, at least one process eventually has completed the detection of all failed processes with lower ID. That process becomes a leader at time $\left(R, t_{4}\right)$ where $t_{4}>t_{3}$ (line 4).

Theorems 1, 2 and Corollary 1 together show that a failure detector of class $Q$ that satisfies Weak Completeness and Strong Accuracy is the weakest failure detector sufficient to solve Election. However, the failure detectors belonging to class $Q$ are strong enough to implement a Perfect Failure Detector, as shown in [10]. Hence, we have the following theorem. 
Theorem 3. A weakest failure detector to solve Election is the Perfect Failure Detector.

PROOF: As we mentioned it above, Theorems 1, 2 and Corollary 1 together show that a failure detector belonging to the class $Q$ is the weakest failure detector sufficient to solve Election. It is shown in [8] that a failure detector of class $Q$ satisfying Strong Accuracy and Weak Completeness can be used to implement a Perfect Failure Detector $P$. Therefore a Perfect Failure Detector is the weakest failure detector that is sufficient to solve Election. This theorem follows from Theorems 1, 2 and Corollary 1.

\section{Concluding Remarks}

The importance of this paper is in extending the applicability field of the results, which Chandra and Toueg have studied on solving problems, into the Election problem in asynchronous system (with crash failures and reliable channels) augmented with unreliable failure detectors.

More specifically, what is the weakest failure detector for solving the Election problem in the asynchronous system? As an answer to this question, we showed that Perfect failure Detector $P$ is the weakest failure detector to solve the Election problem in asynchronous systems. Though $S$ or $W$ are sufficient to solve Consensus, we showed that they are not sufficient to solve Election. Therefore the Election problem is strictly harder than the Consensus problem even when assuming a single crash.

Determining that a problem $\mathrm{Pb}_{1}$ is harder than a problem $\mathrm{Pb}_{2}$ has a very important practical consequence, namely, the cost of solving $\mathrm{Pb}_{1}$ cannot be less than that of solving $\mathrm{Pb}_{2}$. That means that the cost of solving Election cannot be less than that of solving Consensus.

The applicability of these results to problems other than Consensus has been discussed in $[8,17,18,19]$. To our knowledge, it is however the first time that Election problems are discussed in asynchronous systems with unreliable failure detectors. We are not sure that there are problems harder than Election. But we can define failure detectors that are stronger than a Perfect Failure Detector. For example, we can define a failure detector that is not only perfect but also guarantees that a failure of a process is detected only after all messages that it has sent have been received by the detecting process. We can use this failure detector to solve some problems such as the nonblocking version of the asynchronous Primary-Backup problem [12].

\section{References}

1. G. LeLann: Distributed Systems-towards a Formal Approach. Information Processing 77, B. Gilchrist, Ed. North-Holland, 1977

2. H. Garcia-Molina: Elections in a Distributed Computing System. IEEE Transactions on Computers, C-31 (1982) 49-59

3. H. Abu-Amara and J. Lokre: Election in Asynchronous Complete Networks with Intermittent Link Failures. IEEE Transactions on Computers, 43 (1994) 778-788 
4. H.M. Sayeed, M. Abu-Amara, and H. Abu-Avara, "Optimal Asynchronous Agreement and Leader Election Algorithm for Complete Networks with Byzantine Faulty Links.," Distributed Computing, vol. 9, no. 3, pp.147-156, 1995

5. J. Brunekreef, J.-P. Katoen, R. Koymans, and S. Mauw, "Design and Analysis of Dynamic Leader Election Protocols in Broadcast Networks," Distributed Computing, vol. 9, no. 4, pp.157-171, 1996

6. G. Singh: Leader Election in the Presence of Link Failures. IEEE Transactions on Parallel and Distributed Systems, 7 (1996) 231-236

7. M. Fischer, N. Lynch, and M. Paterson: Impossibility of Distributed Consensus with One Faulty Process. Journal of ACM, (32) 1985 374-382

8. T. Chandra and S.Toueg: Unreliable Failure Detectors for Reliable Distributed Systems. Journal of ACM, 43 (1996) 225-267

9. D. Dolev and R Strong: A Simple Model For Agreement in Distributed Systems. In: B. Simons and A. Spector (eds.): Fault-Tolerant Distributed Computing. Lecture Notes in Computer Science, Vol.448. Springer-Verlag, Berlin Heidelberg New York (1987) 42-50

10. T. Chandra, V. Hadzilacos and S. Toueg: The Weakest Failure Detector for Solving Consensus. Journal of ACM, 43 (1996) 685-722

11. Rachid Guerraoui. "Revisiting the Relationship between Non-Blocking Atomic Commitment and Consensus," In Proceedings of the 10th International Workshop on Distributed Algorithms, Springer Verlag (LNCS 857), 1996

12. P.A.Bernstein, V. Hadzilacos, and N. Goodman, "Concurrency Control and Recovery in Database Systems," Addison Wesley, 1987

13. J. E. Hopcroft and J. D. Ullman: Introduction to Automata Theory, Languages and Computation. Addison Wesley, Reading, Mass., 1979

14. Garey M.R. and Johnson D.S: Computers and Intractability: A Guide to the Theory of NPCompleteness. Freeman W.H \& Co, New York, 1979

15. Eddy Fromentin, Michel RAY and Frederic TRONEL: On Classes of Problems in Asynchronous Distributed Systems. In Proceedings of Distributed Computing Conference. IEEE, June 1999

16. Hadzilacos V. and Toueg S: Reliable Broadcast and Related Problems. Distributed Systems (Second Edition), ACM Press, New York, pp.97-145, 1993

17. V. Hadzilacos, "On the Relationship between the Atomic Commitment and Consensus Problems," In Fault-Tolerant Distributed Computing, pp. 201-208. B. Simons and A. spector ed, Springer Verlag (LNCS 448), 1987

18. Schiper and A. Sandoz: Primary Partition: Virtually-Synchronous Communication harder than Consensus. In Proceedings of the 8th Workshop on Distributed Algorithms, 1994

19. R. Guerraoui and A. Schiper: Transaction model vs. Virtual Synchrony model: bridging the gap. In: K. Birman, F. Mattern and A. Schiper (eds.): Distributed Systems: From Theory to Practice. Lecture Notes in Computer Science, Vol. 938. Springer-Verlag, Berlin Heidelberg New York (1995) 121-132 